\title{
Recombinant Rule Selection in Evolutionary Algorithm for Fuzzy Path Planner of Robot Soccer
}

\author{
Jong-Hwan Park ${ }^{1}$, Daniel Stonier ${ }^{2}$, Jong-Hwan Kim², Byung-Ha Ahn ${ }^{1}$, \\ and Moon-Gu Jeon ${ }^{1}$
}

${ }^{1}$ Dept. of Mechatronics, Gwangju Institute of Science and Technology, South Korea

\{jhpark, bayhay, mgjeon\}@gist.ac.kr

${ }^{2}$ Dept. of Electrical Engineering and Computer Science, Korea Advanced Institute of Science and Technology, South Korea

\{stonierd, johkim\}@vivaldi.kaist.ac.kr

\begin{abstract}
A rule selection scheme of evolutionary algorithm is proposed to design fuzzy path planner for shooting ability in robot soccer. The fuzzy logic is good for the system that works with ambiguous information. Evolutionary algorithm is employed to deal with difficulty and tediousness in deriving fuzzy control rules. Generic evolutionary algorithm, however, evaluate and select chromosomes which may include inferior genes, and generate solutions with uncertainty. To ameliorate this problem, we propose a recombinant rule selection method for gene level selection, which grades genes at the same position in the chromosomes and recombine new parent for next generation. The method was evaluated with application of designing the fuzzy path planner, where each fuzzy rule was encoded as a gene. Simulation and experimental results showed the effectiveness and the applicability of the proposed method.
\end{abstract}

\section{Introduction}

To control fast mobile robots, a simple controller is required, which satisfies the mechanical properties such as limitations of wheel speed or translational speed of the robot center. Efficiency of trajectories and short navigation time are also to be ensured. When we consider dribbling and kicking action in robot soccer, robot posture (position and orientation) is of utmost importance. This the paper aims to address the specific problem of robot posture at the target position with emphasis on optimizing the navigational path of the robot.

In the early stages of robot soccer, traditional navigation methods were popular, where the option was to use the simple shortest paths, Dubins path [1] or the composition of rotation, circular motion and straight motion for the path planning step 23. Recently research interest is being focused on the application of fuzzy logic, evolutionary computation, reinforcement learning, unified navigation method, and so on 45677. The use of fuzzy control and behavior-based architectures has been intensively researched in the field of robot navigation, 
because fuzzy logic is a mathematical formulation that copes with uncertainty in information 4 .

However a commonly encountered problem is that the derivation of fuzzy control rules is often time consuming, difficult and relies to a great extent on process experts. An automated way to design fuzzy systems is preferable. In this regard, numerous researches have been dedicated to exploring the use of evolutionary algorithms (EAs) to automate the knowledge acquisition base and construct appropriate rules for a given task 8 81910.

The evolutionary algorithms employed for this purpose use individuals with a single chromosome whose component genes are characterized as rules for the fuzzy control system. During the evolutionary process, a chromosome's performance is tested and the best chromosomes (individuals) are selected for reproduction. A common drawback to this approach is that during testing, some genes (rules) may be seldomly used. These genes contribute negligibly to a global fitness function and consequently the evolutionary process is to a high degree, insensitive to them.

This may lead to a stunted development in the evolution of certain genes as chromosomes are typically selected for reproduction with little dependance on these seldomly used genes. In the worst case scenario it may completely halt development as random processes begin to dominate very slowly evolving genes. In these situations, chromosomes can evolve that have high fitness and perform well for most objectives, but are below par for the few that require the seldomly used genes which have evolved poorly. This causes a variance in the consistency of a chromosome's performance and brings forth uncertainty in the solutions for the real-world problem. These issues are highlighted with an illustrative path planning example in Section 2 .

In this paper, a scheme is formulated that automatically determines the sensitivity of various genes in contributing to the fittest solution. A rule-scoring mechanism ranks genes with the same role (allele) according to their performance under scenarios in which the gene is actually utilized. The parents for the next generation are then formed on the basis of this ranking. The method is then finally trialled on a fuzzy path planner for developing shooting strategies for mobile soccer robots.

This paper is organized as follows. In Section 2 we highlight the difficulties discussed with an illustrative example. Section 3 we present our proposed mechanisms for the evolutionary algorithm. The proposed scheme is analyzed in both simulations and experiments for a robot soccer system in Section 4 Finally, concluding remarks follow in Section 5 .

\section{Illustrative Example - Path Planning}

In this example, the path planning problem for mobile robot navigation is investigated in detail. For this task a path must be planned from a large number of starting points contained in a discretised map of its environment to a specified target. The rule set to be learned is simply a table of desired heading values for 
each point on the discretised map. This rule set is expected to provide a good solution regardless of where the mobile robot is initially placed. This now becomes a multi-objective optimization problem, where each objective is characterized uniquely by the initial conditions, or posture, of the robot relative to the target.

Figure 1illustrates three sample paths generated for a conventionally evolved rule set that has been developed for 48 different initial conditions (starting postures). Here the path planner uses a map of its environment that has been discretised by a fuzzy segmentation of the robot's relative distance and angle from the target. The fitness function used in the evolutionary algorithm is a linear combination of sub-fitness functions for each starting posture and these sub-fitness functions are designed so that the mobile robot approaches its target from the left and in the shortest time possible. For path generation, we also assume the robot uses a controller that adequately tracks the desired heading angle determined by the evolved rule set - this allows us to focus on the path planner.

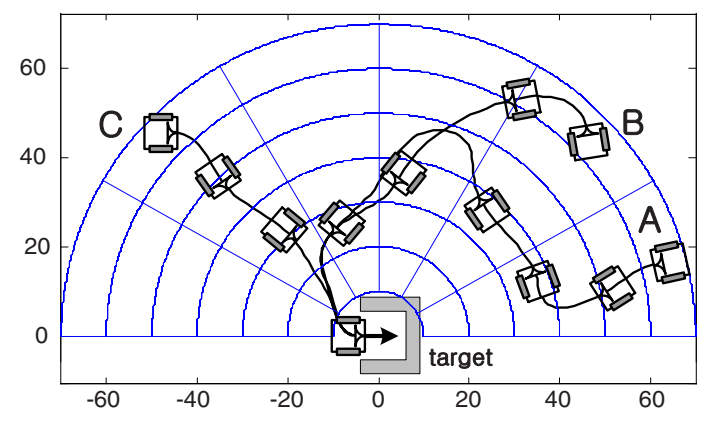

Fig. 1. Comparison of a chromosome's performance for multiple objectives

For this particular scenario, rules that are located in the lower right corner of the figure are utilized by fewer starting postures than rules elsewhere on the map, particularly those immediately to the left of the target. For a uniformly dispersed set of starting postures and a fitness function that weights the performance of each starting posture equally, evolution of these rules may be poor as discussed earlier. This is evident in figure 1 which exhibits reasonably optimal paths for $B$ and $C$ but a less than optimal path for $A$.

These characteristics are highlighted in figure 2 by analyzing the strength and the frequency with which the fuzzy rules are triggered for $A, B$ and $C$ (note that the graph displays the normalized contribution of each fuzzy rule for a particular trajectory - the equations used to generate these firing ratios are presented in Section 3).

For paths $B$ and $C$ fewer rules are triggered - there are many redundancies in the rule space for a particular objective. The rules that initially affect movement from $A$, rules $r_{1}$ to $r_{12}$ (shaded grey in figure 2), have evolved poorly since very few starting postures require them. Subsequently these contribute negligibly to the global fitness function. They do not provide a very good solution for $A$, but 


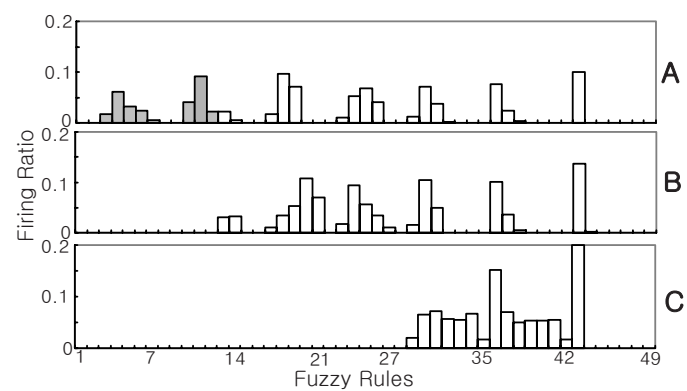

Fig. 2. Firing ratios of fuzzy rules for $\mathrm{A}, \mathrm{B}$ and $\mathrm{C}$

they are kept since the fuzzy rule set (chromosome) in general performs very well. Note that the evolved rule set here provides very good directions in the immediate vicinity to the left of the target - this is to be expected as almost every starting posture must utilize these rules for a successful approach. As a result the global fitness function will be highly sensitive to these rules and they evolve both rapidly and accurately.

In order to ensure improved evolution for these underdeveloped rules, a means for determining their sensitivity in the evolutionary process is needed as well as an alternate method ranking the performance of the chromosomes.

\section{Proposed Evolutionary Technique}

The conventional evolutionary algorithm used for the illustrative example utilized a selection scheme based on a $q$-tournament. A $(\mu, \lambda)$-evolution strategy [11] was used and the elite chromosome saved by the elitist strategy [12]. Here, various modifications are proposed for the parent selection process that assist in the evolution of optimal solutions for multi-objective path planning problems.

\subsection{Parent Selection Process}

For a highly redundant problem, a gene (rule) may only be a necessary parameter for the optimization of a small portion of the objectives. Consequently evolving a gene for a more globally optimal solution by considering the performance of objectives that do not utilize it is redundant. It introduces complexity and in the course of an evolutionary algorithm, this will slow or halt its evolution.

The usual process of parent selection in an evolutionary algorithm is to find the best chromosome for all genes when tested against all objectives simultaneously. Our approach is to develop a unique scoring method that finds the highest ranked chromosomes for each gene when tested against only the objectives that utilize that gene. We then form the parents for the next generation from this pool of chromosomes. Some important aspects of the process are as follows: 
- The method reverts to a conventional parent selection process if there is no redundancy.

- The final solution will converge more rapidly for systems with redundancy.

- Where evolution of particular genes comes to a halt, increasing the rate of gene evolution with this approach may provide an improvement in the final solution.

\subsection{Implementation}

Implementing this method in an evolutionary algorithm can be broken down into the following steps:

- Determining and prioritizing an objective's dependence on a particular gene.

- Scoring method to find a gene's 'best' chromosome.

- Forming the parents for the next generation.

Gene-Dependence. Given a chromosome and an objective, an objective's dependence on a particular gene can be found by prioritizing the frequency and strength with which the fuzzy rule is triggered as a solution is generated for the chromosome-objective pair. This is a process which is also gradually learned by the evolutionary algorithm as chromosomes evolve. Figure 2 illustrated the concept in the introductory example.

For the navigation problem, path generation is broken down into path planning and path following operations that are performed at discrete time intervals. At each step, the chromosome is used to determine the genes that are triggered and generate a desired path to follow. The path following controller is used to track the desired path until the next time interval at which point the process is repeated. By combining these discrete steps, a path is generated.

The mechanism for determining the strength and frequency with which rules are triggered along these paths is presented as follows. Discrete time steps are defined by $t_{i}, i=0 \ldots n$ where $t_{0}=0$ and $t_{n}$ is the elapsed time taken for the path to terminate. At the $i$-th step on the path generated for the $j$-th objective (starting posture) using the $k$-th chromosome, we collect the normalized firing strength of the $l$-th gene, the weight of each rule for center average defuzzifier [13, is defined by

$$
N F S_{i, j, k, l}=\left.\frac{w_{j, k, l}}{\sum_{m} w_{j, k, m}}\right|_{t_{i}},
$$

where $w_{j, k, l}$ is the strength with which the $l$-th gene (fuzzy rule) is triggered. The total firing strength of the $l$-th gene over the whole path is then

$$
F S_{j, k, l}=\sum_{i=1}^{n} N F S_{i, j, k, l} .
$$

The firing ratio for the $l$-th gene on this path is simply the firing strength normalized for the firing strength of all genes triggered on this path. This is defined by 


$$
F R_{j, k, l}=\frac{F S_{j, k, l}}{\sum_{m} F S_{j, k, m}} .
$$

It is the firing ratio of each gene for a particular chromosome and starting posture that can be seen graphically represented on the bar graph in figure 2 .

Scoring Method. The next step is to rank chromosomes for each gene in a prioritized order to find a gene's 'best' chromosome. For this we define the Rule Score of the k-th chromosome for the l-th gene with

$$
R S_{k, l}=\sum_{j}\left(F R_{j, k, l} \times C W_{j, k}\right),
$$

where $C W_{j, k}$ is the count of wins for the $k$-th chromosome by q-tournament for the $j$-th objective (starting posture). Note that if an objective does not have any dependence on a gene, it will not contribute to the rule score for that gene since its firing ratio will be zero. It also works more effectively than a simple test for each chromosome as it weights results according to the objective's dependence on the gene.

Forming the Parents. The final step is to assemble the parents ( $\mu$ parents) for the next generation from the existing group of highly ranked individuals $(\lambda$ chromosomes). The first parent is selected by the elitist strategy as the fittest individual for the global solution. The remainder are formed by recombining chromosomes at the gene level. The genes for a chromosome are ranked on the basis of their score amongst others in the same column (allele) of chromosomes. The genes of the first rank in each position, $h$, are collected into the first chromosome, $R_{1}^{\prime}$. The genes of the second rank form a second chromosome, $R_{2}^{\prime}$ and so forth. A single instance of this process is illustrated in figure 3 . From these, an extended family of parent chromosomes is formed that disperses itself through the search space in a manner that allows solutions for seldomly triggered genes (rules) to be more readily found.

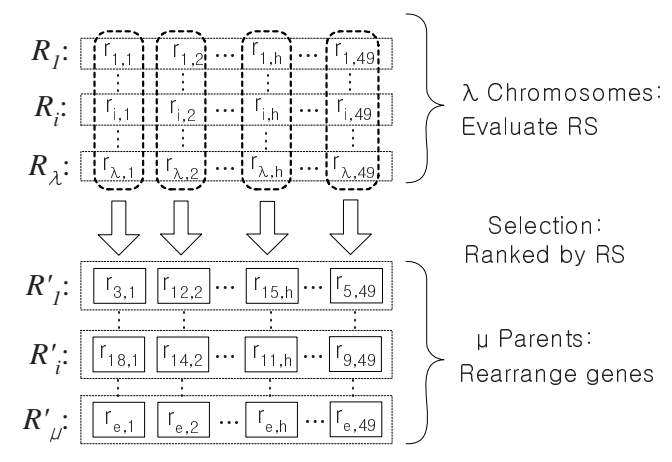

Fig. 3. Reproduction with recombinant rule selection 


\section{Experiments}

\subsection{Robot Soccer System}

To demonstrate the effectiveness and applicability of the proposed method, a fuzzy evolutionary system was developed for enhancing the performance of mobile robot behaviours in the MIROSOT soccer system [141516]. Behaviours for the Mirosot system that can be enhanced with the approach presented in this paper include passing, defending and shooting. In this experiment the path planning approach is applied for improving the speed, reliability and consistency of the shooting behaviour. Localisation of the robots in the MIROSOT system is achieved via an overhead vision system connected to an external PC that develops strategies and controls which are then transmitted to the robots on the playing field.

\subsection{Fuzzy System}

The fuzzy system that was developed is comprised of two modules connected in hierarchical fashion, a fuzzy path planner and a fuzzy path-following controller [17,18. This is illustrated in figure 4. The fuzzy path planner is responsible for generating the desired paths from the initial posture to the ball position that are optimized for various shooting performance criteria and also satisfy any necessary constraints. It is again assumed the fuzzy controller can adequately track the desired heading angle so that the focus remains on the fuzzy path planner.

The fuzzy planner is designed to accept fuzzified information describing the mobile robot's relative position with respect to the ball $(\rho, \varphi)$ as inputs for a set of fuzzy rules that determine the appropriate desired heading angle $\theta_{D}$ corresponding to each input. These fuzzy inputs are used to discretise the map

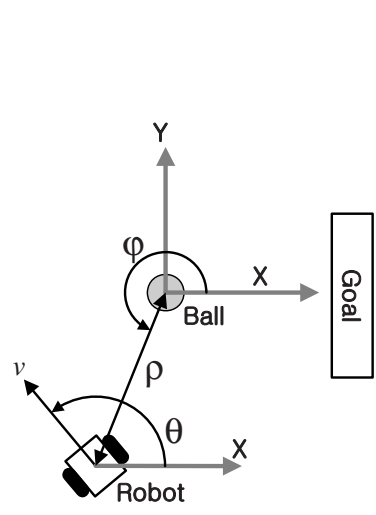

(a)

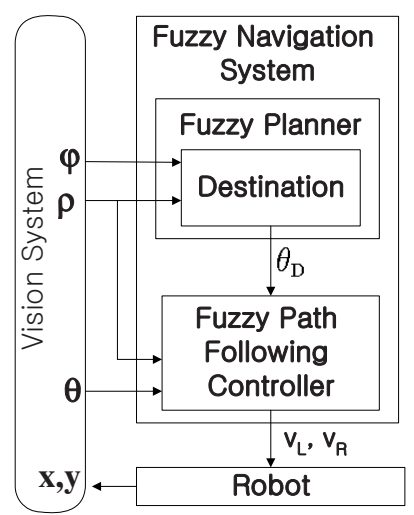

(b)

Fig. 4. Overall structure of fuzzy navigation system (a) Localisation variables (b) Fuzzy navigation system 
of the robot's environment and the rules are used to generate paths consisting of singleton values for the direction at sampled positions for a trajectory to the ball, like a univector field 1920.

\subsection{The Evolutionary Algorithm}

An evolutionary algorithm with the modifications presented in Section 3 is used to learn the fuzzy rules. The algorithm uses a $(\mu, \lambda)$-strategy where the number of parents $(\mu)$ and offspring $(\lambda)$ is set to 10 and 20, respectively. The $q$-tournament selects 10 competitors in each round.

Each chromosome in the algorithm represents an entire fuzzy rule set and each gene represents an individual fuzzy rule mapping the inputs $(\rho, \varphi)$ to the output $\theta_{D}$. Inputs are constrained to $0 \mathrm{~cm} \leq \rho \leq 80 \mathrm{~cm}$ and $0^{\circ} \leq \varphi \leq 180^{\circ}$ (there exists a geometrical symmetry for the simple case containing no obstacles). A typical chromosome is illustrated in Table 1 where each input variable is spanned by seven membership functions (here the fuzzified $\rho$ values range from very near to very far and the angle $\varphi$ from very small to very large). Subsequently, there are in total 49 genes within the chromosome for this experiment.

Table 1. Rules for desired heading angle

\begin{tabular}{c|ccccccc}
\hline$\theta_{D}$ & \multicolumn{6}{|c}{$\rho$} \\
\hline$\varphi$ & VN & AN & SN & MD & SF & AF & VF \\
\hline VS & 11 & 144 & 119 & 125 & 76 & 74 & 206 \\
LS & 36 & 121 & 151 & 189 & 213 & 200 & 170 \\
SS & 315 & 169 & 231 & 234 & 203 & 239 & 242 \\
MD & 335 & 199 & 212 & 255 & 238 & 254 & 237 \\
SL & 153 & 268 & 269 & 267 & 301.7 & 276 & 295 \\
AL & 296 & 305 & 315 & 334 & 346.1 & 276 & 350 \\
VL & 6 & 344 & 351 & 349 & 332.3 & 335 & 331 \\
\hline
\end{tabular}

Solutions to the evolutionary algorithm are required to be successful whilst minimizing for elapsed time $t_{l}$ and vertical drift/orientation errors, $y_{e}, \theta_{e}$. The $\mathrm{x}$-axis represents the desired heading direction at the target. These variables are considered to be the necessary performance criteria for shooting and are illustrated in figure 5 .

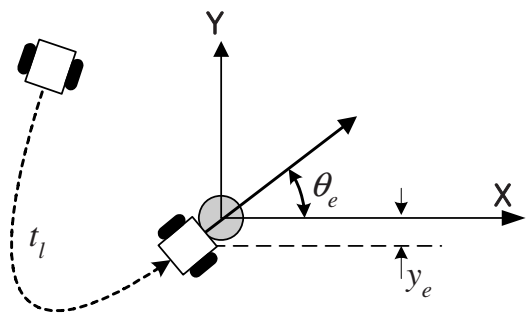

Fig. 5. Performance factors for shooting ability 
To evaluate the fitness of the $i$-th chromosome for the $j$-th objective (initial posture), the performance index (PI) is defined as

$$
P I_{i, j}=K_{t} \cdot t_{l}+K_{p} \cdot\left|\theta_{e}\right|+K_{d} \cdot y_{e}^{2},
$$

where $K_{t}, K_{p}$ and $K_{d}$ are positive constance. The performance index of the $i$-th chromosome for a group, $\Sigma$ of objectives is simply defined as the cumulative sum of its performance indexes for each objective:

$$
P I_{i}=\sum_{j \in \Sigma} P I_{i, j}
$$

This is used in both the $q$-tournament and as a tool to evaluate the fitness of chromosomes once the algorithm has completed. The coefficients were manually tuned and finalized at $K_{t}=10, K_{p}=1$ and $K_{d}=3$. These provided almost equal weightings for each criteria in the performance index - that is $K_{t} \cdot t_{l} \approx$ $K_{p} \cdot\left|\theta_{e}\right| \approx K_{d} \cdot y_{e}^{2}$ for nearby solutions in this experiment.

Forty-eight points (initial postures) were selected for an exercise in which all the genes were used at least once. The evolutionary algorithm was evolved for 3,000 generations in both the conventional and proposed rule-scoring methods. To compare results, the evolutionary algorithm was applied using both conventional and proposed scoring methodologies 62 times each. Performance index values were recorded during evolution and utilized to calculate statistical data. For the statistical analysis, consistency of the results was also defined as an important measure of the reliability of the evolved chromosome as a solution. For this, the coefficient of variation $(\mathrm{CV})$ was used since it provides a degree of invariance for comparing solutions with different initial postures and travel lengths. The coefficient of variation for a variable $x$ is defined as

$$
C V(x)=\frac{\operatorname{Std}(x)}{\operatorname{Mean}(x)} \times 100 \quad(\%) .
$$

Table 2 shows the simulation results for each method based on the overall performance index of the evolved rule set. Clearly the proposed method performs better with a smaller mean value and has a standard deviation less than the one-third that of the conventional method. It also shows a decrease in the co-efficient of variation of the PI for individual starting postures (figure 6) and exhibits faster convergence (figure 7) than the conventional method. Moreover, the coefficient of variation was reduced by about $50 \%$ on average, and the coefficient of variation of the performance index for each objective (training point or

Table 2. Converged global performance index

\begin{tabular}{c|cc} 
Algorithm & Mean & Std \\
\hline Conventional selection & 434.39 & 45.88 \\
Recombinant selection & 382.51 & 15.22 \\
\hline
\end{tabular}




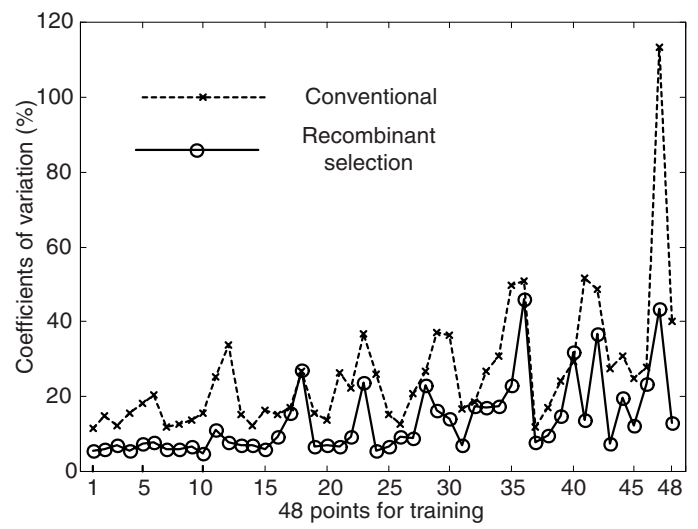

Fig. 6. Coefficient of variation of PI for individual training points

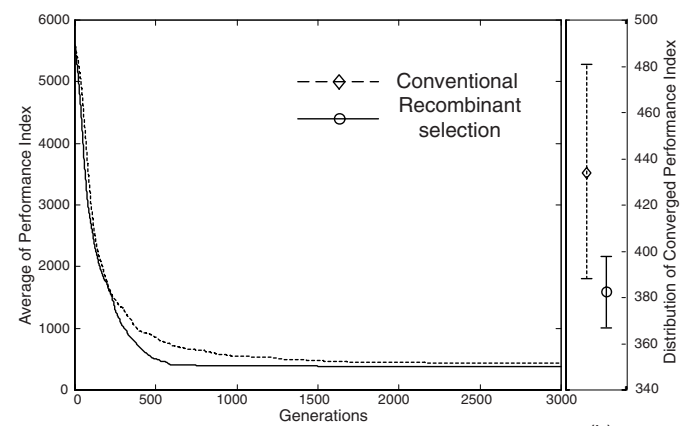

(a) (b)

Fig. 7. Comparison of global performance index (a) Convergence (b) Variance

initial condition) also improved evenly (figure 6). These results imply a tendency for the proposed algorithm to consistently find more optimal solutions than the conventional method.

\subsection{Experiment Results}

The applicability of the proposed method was physically tested on a mobile robot where an improvement in shooting ability was desired. From the 62 fuzzy rule sets generated by both conventional and proposed methods, 27 were randomly selected for testing of the robot's shooting ability. The robot was initialized from five distinct postures with various pre-specified facings. To compensate for variation from the noise caused by physical disturbances and errors, the robot was tested five times for each combination of starting posture and applied rule set.

The elapsed traveling time and the direction of the ball in motion were used to evaluate path effectiveness. The direction of the ball (shooting angle error) was used to represent the effects of both vertical drift and heading angle errors 
as these were individually difficult to measure reliably. A statistical analysis is shown in Tables 3 and 4 . The results indicate the proposed rule-scoring method consistently generates paths that have shorter elapsed times with significantly reduced variation (CV). These satisfied the original goals (speed and improved reliability of shooting) whilst maintaining a heading angle error reasonable for the dimensions of the field. Improved heading angle error can be achieved by a suitable tuning of the weightings in the Performance Index function.

Table 3. Elapsed time for shooting in experiments (sec)

\begin{tabular}{|c|c|c|c|c|c|c|}
\hline \multirow{2}{*}{$\begin{array}{l}\text { Start } \\
\text { point }\end{array}$} & \multicolumn{3}{|c|}{ Conventional selection } & \multicolumn{3}{|c|}{ Recombinant selection } \\
\hline & Mean & Std & $\mathrm{CV}(\%)$ & Mean & Std & $\mathrm{CV}(\%)$ \\
\hline$\overline{\mathrm{A}}$ & 2.60 & 0.33 & 12.61 & 2.33 & 0.14 & 6.00 \\
\hline B & 2.23 & 0.28 & 12.71 & 2.00 & 0.11 & 5.60 \\
\hline $\mathrm{C}$ & 1.93 & 0.28 & 14.46 & 1.71 & 0.10 & 5.60 \\
\hline D & 1.73 & 0.23 & 13.63 & 1.60 & 0.06 & 3.61 \\
\hline $\mathrm{E}$ & 1.77 & 0.22 & 12.45 & 1.59 & 0.07 & 4.09 \\
\hline Average & 2.05 & 0.27 & 13.14 & 1.85 & 0.09 & 4.98 \\
\hline
\end{tabular}

Table 4. Shooting Angle error for shooting in experiments (deg)

\begin{tabular}{|c|c|c|c|c|c|c|}
\hline \multirow{2}{*}{$\begin{array}{l}\text { Start } \\
\text { point }\end{array}$} & \multicolumn{3}{|c|}{ Conventional selection } & \multicolumn{3}{|c|}{ Recombinant selection } \\
\hline & Mean & Std & $\mathrm{CV}(\%)$ & Mean & Std & $\mathrm{CV}(\%)$ \\
\hline$\overline{\mathrm{A}}$ & 5.11 & 3.72 & 72.78 & 6.46 & 4.24 & 65.75 \\
\hline B & 4.67 & 3.45 & 73.98 & 6.26 & 3.01 & 48.19 \\
\hline C & 4.41 & 3.19 & 72.19 & 6.20 & 3.46 & 55.78 \\
\hline D & 4.68 & 3.13 & 66.74 & 6.11 & 3.23 & 52.87 \\
\hline $\mathrm{E}$ & 3.95 & 2.62 & 66.24 & 5.28 & 3.66 & 69.21 \\
\hline Average & 4.36 & 3.22 & 70.20 & 6.06 & 3.52 & 58.8 \\
\hline
\end{tabular}

Figure 8 illustrates several shooting solutions generated by both conventional and proposed methods. Figure 8(a) shows several trajectories generated by an applied rule set derived using the conventional method. As discussed earlier, chromosomes that performed well (relatively low PI) were selected to evolve the population. These often had genes (rules) that were triggered for a select few paths on which they performed poorly. Consequently evolution of these genes did not occur and the final rules needed for these poorly evolved points remained inferior. This is clearly seen in the figure where paths generated for D and E provide successful solutions, however the remaining paths for $\mathrm{A}, \mathrm{B}$ and $\mathrm{C}$ deviate undesirably.

The proposed rule-scoring method discriminates among genes using the strength of the rules. This eliminates inferior rules and allows for uniform evolution of rules across the entire input space. This is highlighted in figure 8(b).

It is worth noting that this procedure can be used to derive the fuzzy path following controller (refer to figure 4) by determining wheel velocities given a relative heading angle error and the radial distance from the target as the inputs. 


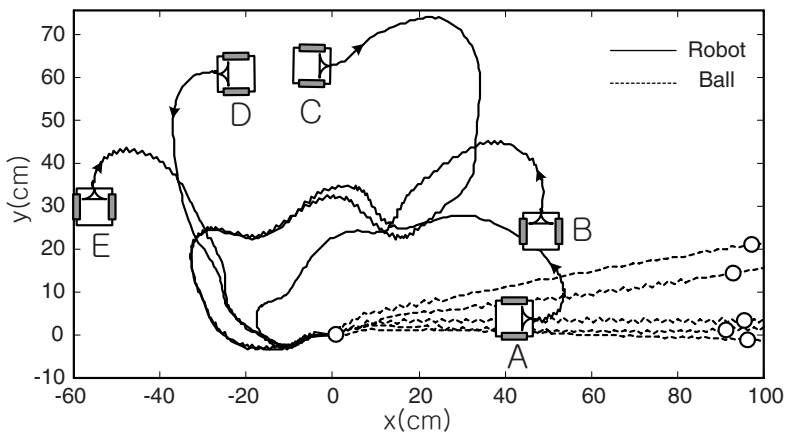

(a) conventional method

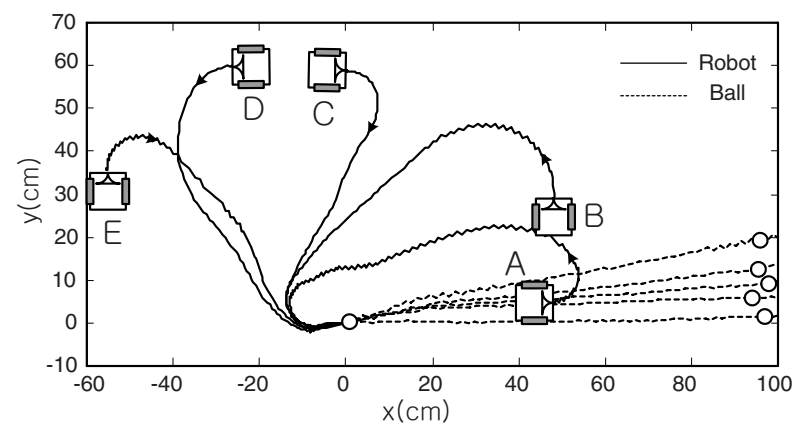

(b) Proposed method

Fig. 8. Experiment of robot shooting

This problem exhibits the same properties as the path planner and the proposed algorithm can assist in learning of the seldomly triggered rules.

\subsection{Experimental Conclusions}

One of the most important features of the path planning problem demonstrated here is that there exists an optimal solution that is shared by all objectives (consider two points in line with the target - the rule set for the rearmost point is equally valid for the closer point). This property is typical of such path planning problems in general. In these situations, the rule-scoring method helps identify chromosomes that perform well for seldomly triggered rules and the gene recombination used to form parents for the next generation more aggressively directs the solution to progress towards the common goal.

For more complex nonlinear optimization problems with opposing objectives, a pareto optimal solution must be found and an alternative process may be needed for forming the non-elite parents of the next generation. Stochastic contributions may be necessary to ensure gene recombination do not consistently and adversely affect each other as they strive toward differing goals. This avenue is currently being explored by the authors and is open to further research. 


\section{Conclusion}

Conventional evolutionary algorithms for multi-objective problems evolve genes by ranking chromosomes for the reproduction process based on their performance on all objectives simultaneously. This often results in poorly evolved rules for those rules which are needed by only a few objectives. In this paper, new methods are proposed for the mobile robot path planning problem which prioritize objectives that utilize a particular gene and rank chromosomes against these objectives on a gene by gene basis. The reproduction process then selects the information from the best of each of these groups and rearranges the rules for reproducing the parents of the next generation. This enables the seldomly triggered rules to evolve at a much higher rate. Experimental results on a path planning problem in robot soccer verifies these results. Solutions evolved with the proposed method were faster and most importantly they exhibited a higher consistency of performance than was possible using a conventional method.

\section{Acknowledgement}

This research is supported by the Ubiquitous Computing and Network (UCN) Project, the Ministry of Information and Communication (MIC) 21st Century Frontier R\&D Program in Korea.

\section{References}

1. L. E. Dubins: On curves of minimal length with a constraint on average curvature, and with prescribed initial and terminal positions and tangents. Amer. J. Math., 79, (1957) 497-516

2. J.-H. Kim, H.-S. Shim, H.-S. Kim, M.-J. Jung, I.-H. Choi, and J.-O. Kim: A cooperative multi-agent system and its real time application to robot soccer. IEEE Proc. Int. Conf. Robot. Automat. Albuquerque, NM, (1997)638-643

3. W.-M. Shen et al: Building integrated mobile robots for soccer competition. IEEE Proc. Int. Conf. Robot. Automat. vol. 3, Leuven, Belgium, May, (1998) 2613-2618

4. M.-J. Jung H. S. Kim, H. S. Shim, and J. H. Kim: Fuzzy rule extraction for shooting action controller of soccer robot. IEEE Proc. Int. Conf. Fuzzy Syst. 1, (1999) 556-561

5. D.-H. Kim et al.: Vector field based path planning and Petri-net based role selection mechanism with Q-learning for the soccer robot system. Intell. Automat. Soft Comput. In: J.-H. Kim et al. (eds)., 6, (2000) 75-87

6. W.-G. Han et al.: GA based on-line path planning of mobile robots playing soccer games. Proc. IEEE 40th Midwest Symp. Circuit Syst. Sacramento, CA, Sept.(1998) $522-525$

7. J.-H. Kim et al.: Path planning and role selection mechanism for soccer robots. Proc. IEEE Int. Conf. Robot. Automat. Vol. 4. Leuven, Belgium, (1998) 32163221

8. F. Hoffmann: Evolutionary Algorithms for Fuzzy Control System Design. Proceedings of the IEEE, 89 (9) (2001) 1318-1333. 
9. S.-J. Kang, C.-H. Woo, H.-S. Hwang, K.B. Woo: Evolutionary design of fuzzy rule base for nonlinear system modeling and control. IEEE Transactions on Fuzzy Systems. 8 (1) (2000), $37-45$.

10. D.-H. Park, A. Kandel: Genetic-Based New Fuzzy Resoning Models with Application to Fuzzy Control. IEEE Transactions on Sys., Man, and Cybernetics. 24(1) (1994), 39-47

11. H.-P. Schwefel: Numerical Optimization of Computer Models. UK:John Wiley, Chichester, (1981)

12. D. E. Goldberg: Genetic Alogrithms in Search, Optimization, and Machine Learning. Addison-Wesley (1989)

13. Li-Xin Wang: A Course in Fuzzy Systems and Control. Prentice Hall(Int. ed.). (1997) 110

14. J.-H. Kim, (ed): Robotics and Autonomous Systems. Special Issue: First MicroRobot World Cup Soccer Tournament, MiroSot, 21(2)(1997).

15. J.-M. Yang and J-H. Kim: Sliding Mode Control for Trajectory Tracking of Nonholonomic Wheeled Mobile Robots. IEEE Transactions on Robotics and Automation. 15(3) (1999), 578-587.

16. J.-H. Kim, D.-H. Kim, Y.-J. Kim, K.-T. Seow, Soccer Robotics (Springer Tracts in Advanced Robotics), Springer Verlag, 3540218599, 326, 2004.

17. M.-J. Jung, H.-S. Shim, H.-S. Kim, J.-H. Kim: Fuzzy rule extraction for shooting action controller of soccer robot. Fuzzy Systems Conference Proceedings. 1 (1999), $556-561$.

18. M.-S. Lee, M.-J. Jung, J.-H. Kim: Evolutionary programming-based fuzzy logic path planner and follower for mobile robots. Proceedings of the 2000 Congress on Evolutionary Computation. 1 (2000), 139 - 144

19. Y.-J. Kim, J.-H. Kim and D.-S. Kim: Evolutionary Programming-Based Uni-vector Field Navigation Method for Fast Mobile Robots. IEEE Trans. on Systems Man and Cybernetics - Part B - Cybernetics. 31(3) (2001) 450-458

20. M. Mizumoto: Fuzzy controls by fuzzy sington-type resoning method. Proc. of the Fifth IFSA world congress. Seoul, Korea, (1993) 945-948 